\title{
Prognostic and predictive value of circulating tumor cells and CXCR4 expression as biomarkers for a CXCR4 peptide antagonist in combination with carboplatin-etoposide in small cell lung cancer: exploratory analysis of a phase II study
}

\author{
Ravi Salgia $^{1} \cdot$ R. Waide Weaver ${ }^{2} \cdot$ Michael McCleod $^{3} \cdot$ John R. Stille $^{4} \cdot$ S. Betty Yan $^{5}$. \\ Stephanie Roberson ${ }^{4} \cdot$ John Polzer $^{4}$ • Amy Flynt ${ }^{6} \cdot$ Eyas Raddad $^{4} \cdot$ Victoria L. Peek ${ }^{5}$. \\ Sameera R. Wijayawardana ${ }^{5}$ - Suzane L. Um ${ }^{5} \cdot$ Steve Gross $^{7} \cdot$ Mark C. Connelly ${ }^{7}$. \\ Carrie Morano $^{7} \cdot$ Madeline Repollet $^{7} \cdot$ Renouard Sanders $^{7} \cdot$ Kurt Baeten $^{8}$. \\ David D'Haese $^{8} \cdot$ David R. Spigel $^{9}$
}

Received: 19 December 2016 / Accepted: 23 February 2017 / Published online: 15 March 2017

(C) The Author(s) 2017. This article is published with open access at Springerlink.com

Summary Background Circulating tumor cells (CTCs) and chemokine (C-X-C motif) receptor 4 (CXCR4) expression in CTCs and tumor tissue were evaluated as prognostic or predictive markers of CXCR4 peptide antagonist LY2510924 plus carboplatin-etoposide (CE) versus CE in extensivestage disease small cell lung cancer (ED-SCLC). Methods This exploratory analysis of a phase II study evaluated CXCR4 expression in baseline tumor tissue and peripheral blood CTCs and in post-treatment CTCs. Optimum cutoff values were determined for CTC counts and CXCR4

Electronic supplementary material The online version of this article (doi:10.1007/s10637-017-0446-z) contains supplementary material, which is available to authorized users.

Ravi Salgia

rsalgia@coh.org

1 City of Hope Comprehensive Cancer Center, 1500 E. Duarte Road, Duarte, CA 91010-3000, USA

2 Florida Cancer Specialists, St. Petersburg, FL, USA

3 Florida Cancer Specialists, Fort Myers, FL, USA

4 The Chorus Group, Eli Lilly and Company, Indianapolis, IN, USA

5 Lilly Research Laboratories, Eli Lilly and Company, Indianapolis, IN, USA

6 PharPoint Research Inc., Durham, NC, USA

7 Janssen Diagnostics, Johnson and Johnson Company, Raritan, NJ, USA

8 Janssen Diagnostics, Janssen Pharmaceutica, Beerse, Belgium

9 Sarah Cannon Research Institute, Nashville, TN, USA expression in tumors and CTCs as predictors of survival outcome. Kaplan-Meier estimates and hazard ratios were used to determine biomarker prognostic and predictive values. Results There was weak positive correlation at baseline between CXCR4 expression in tumor tissue and CTCs. Optimum cutoff values were $\mathrm{H}$-score $\geq 210$ for $\mathrm{CXCR} 4^{+}$tumor, $\geq 7 \%$ CTCs with CXCR4 expression (CXCR $4^{+}$CTCs), and $\geq 6$ CTCs/7.5 mL blood. Baseline $\mathrm{H}$-score for CXCR4 ${ }^{+}$tumor was not prognostic of progression-free survival (PFS) or overall survival (OS). Baseline CXCR $4^{+} \mathrm{CTCs} \geq 7 \%$ was prognostic of shorter PFS. CTCs $\geq 6$ at baseline and cycle 2 , day 1 were prognostic of shorter PFS and OS. None of the biomarkers at their respective optimum cutoffs was predictive of treatment response of LY2510924 plus CE versus CE. Conclusions In patients with ED-SCLC, baseline CXCR4 expression in tumor tissue was not prognostic of survival or predictive of LY2510924 treatment response. Baseline $\mathrm{CXCR}^{+}{ }^{+} \mathrm{CTCs} \geq 7 \%$ was prognostic of shorter PFS. CTC count $\geq 6$ at baseline and after 1 cycle of treatment were prognostic of shorter PFS and OS.

Keywords LY2510924 · CXCR4 expression · Circulating tumor cells · Small cell lung cancer · Carboplatin-etoposide

\section{Introduction}

Small cell lung cancer (SCLC) accounts for approximately $10-15 \%$ of all lung cancer cases and is characterized by aggressive growth, early development of metastases, high mortality, and initial response to chemotherapy followed by 
relapse [1]. Approximately $60-70 \%$ of patients with SCLC have extensive-stage disease (ED) [2]. The 5year survival rate for patients with SCLC is only $7 \%$ [3]. Patients with ED-SCLC have a median survival of 7-11 months with currently available therapy, and longterm disease-free survival is rare [4]. Despite development of targeted therapies for non-small cell lung cancer (NSCLC), there have been few advances in treatment options for patients with SCLC.

To date, no predictive validated biomarker is available for SCLC outcomes. A meta-analysis showed that elevated chemokine (C-X-C motif) receptor 4 (CXCR4) expression correlated with shorter overall survival (OS) in patients with NSCLC and suggested a poor prognostic outcome of this disease [5]. The value of CXCR4 expression as a prognostic marker of SCLC is not known. A lack of preclinical models or access to patient tissues samples, as surgery is rarely used to treat SCLC, yields few tumor specimens for research. Tumor biopsies of SCLC are difficult to obtain, frequently with low tumor purity, and the tissue is often necrotic [6]. Repeat biopsies are even more problematic, which compounds the challenges.

Collection of circulating tumor cells (CTCs) permits evaluation of biomarkers such as CXCR4 expression in patients with SCLC, as CTCs are present in high numbers in the peripheral blood of patients with SCLC [7-9]. This facilitates using CTCs as a liquid biopsy to examine genetic or phenotypic markers in patients with SCLC, and provides an opportunity for repeated sampling during the course of treatment. In addition to evaluating CXCR4 expression in CTCs, recent reports have suggested that CTCs alone may be a useful prognostic or predictive factor for survival in patients with SCLC, both at baseline and following 1 or 2 cycles of chemotherapy [10-12]. Cheng et al. [13] reported that, following a second cycle of treatment, CTCs and change in CTC counts from baseline were independent indicators for both progression-free survival (PFS) and OS in patients with ED-SCLC. In a pilot study analyzing CTCs as a biomarker of chemotherapy response and relapse, higher baseline CTCs in treatment-naïve patients with ED-SCLC and the percentage of decrease in posttreatment CTCs were associated with decreased survival [14].

CXCR4 is implicated in tumor cell motility, survival, and growth and is often overexpressed in SCLC tumors and SCLC cell lines [15-17]. CXCR4 activation in SCLC has been shown to induce migration and invasion into the extracellular matrix; marrow stromal cells or extracellular matrix proteins may protect SCLC cells from chemotherapy-induced apoptosis [15, 18, 19], suggesting a role for CXCR4 in chemotherapy resistance. Stromal cellderived factor-1 (SDF-1, C-X-C motif chemokine 12) is an important ligand of CXCR4 [20].
A phase II study of LY2510924, a novel cyclic peptide that blocks the binding of the ligand SDF-1 (CXCL12) to CXCR4 $[16,21]$, was conducted in patients with ED-SCLC who received LY2510924 plus carboplatin-etoposide (CE) versus CE alone (NCT01439568) [22]. There was no difference in median PFS, the primary efficacy endpoint, between the treatment arms in this study [23], which was approximately 5.9 months in both arms. In exploratory analyses of patients with ED-SCLC and high CXCR4-expressing tumors at baseline (H-score $\geq 210$ ), the hazard ratio (HR) for PFS between the treatment arms was 0.787 (95\% confidence interval [CI]: 0.211, 2.933) [24]. Thus, CXCR4 overexpression in SCLC tumors does not appear to be a predictive biomarker for treatment response to LY2510924 plus CE versus CE alone. Here, the aim of our post-hoc exploratory analyses was to evaluate the potential prognostic value of CTC counts and CXCR4 expression in CTCs and in tumor tissue in the overall study population, and to evaluate potential predictive value of these biomarkers for treatment with LY2510924 plus CE versus CE alone. In addition, we evaluated the correlation of baseline CXCR4 expression in tumor tissue and in CTCs from patients in this phase II study.

\section{Methods}

\section{Patients and treatments}

In a phase II study [22], patients with ED-SCLC were randomized 1:1 to receive CE versus LY2510924 plus CE. Carboplatin (area under the curve [AUC] 5) and etoposide $\left(100 \mathrm{mg} / \mathrm{m}^{2}\right)$ were administered intravenously on day 1 and days 1-3, respectively, for up to six 21-day cycles. LY2510924 (20 mg) was administered subcutaneously daily on days 1-7 for up to six 21-day cycles. In accordance with the Declaration of Helsinki, written informed consent was obtained from all enrolled patients before initiating any study procedure. The study protocol was approved by an ethics committee and conformed to principles of Good Clinical Practice.

\section{Isolation and enumeration of CTCs and detection of CXCR4 expression}

Blood from patients with ED-SCLC was collected into a CellSave preservative tube (Janssen Diagnostics, Raritan, NJ) at baseline (day 1 of cycle 1), day 7 of cycle 1 , day 1 of cycle 2, and at 30-day follow-up after the last dose of study drug. Circulating tumor cells were isolated and enumerated using the CXC CELLSEARCH® kit and CELLSEARCH® system according to the manufacturer's instructions (Janssen Diagnostics LLC, Raritan, NJ) as previously described [25]. Details of the CTC assay for CXCR4 expression detection are 
provided in Supplementary Materials and Methods. After CTC enumeration of each sample, CXCR4 expression was determined by an operator and defined as CXCR4-positive or -negative by visual phenotyping and classification. Details of CXCR4 quantification are provided in the Supplementary Materials and Methods. Representative images of tumor cell lines expressing varied levels of CXCR4 and a CXCR4+ CTC sample from a patient with SCLC are shown in Supplementary Fig. S1a-g.

\section{Baseline CXCR4 expression in tumors by immunohistochemistry}

CXCR4 expression analysis was performed on formalinfixed, paraffin-embedded tumor tissue. Immunohistochemistry (IHC) staining for CXCR4 (rabbit anti-human CXCR4 monoclonal antibody, clone UMB2; Epitomics Inc., Burlingame, CA) was performed at Bostwick Laboratories (Uniondale, NY) on an intelliPATH FLX AutoStainer (Biocare Medical, Concord, CA) using heat-induced antigen retrieval and standard techniques with 3,3'-diaminobenzidine as chromogen and hematoxylin counterstain. Stained slides were reviewed by board-certified pathologists; an H-score (0-300) for CXCR4 expression was assigned to each sample. Immunostaining intensity was assessed by visual assessment: 0 (no staining), 1+ (low intensity), 2+ (intermediate intensity), and 3+ (strong intensity). $\mathrm{H}$-scores were calculated from the percentage of cells (in increments of 10\%) at different staining intensities, using the formula: 0 ( $\%$ of 0 staining cells $)+$ $1 \times(\%$ of $1+$ staining cells $)+2 \times(\%$ of $2+$ staining cells $)+3 \times(\%$ of $3+$ staining cells).

\section{Statistical analysis methods}

A 2-sided significance (alpha) level of 0.05 was used to identify variables that may be predictive of PFS at 6 months or OS at 11.5 months. All other statistical testing was 2-sided at alpha level of 0.10 . These analyses were hypothesesgenerating and no adjustments for multiple comparisons were made; results should be interpreted with caution due to the possibility of a false positive. All statistical analyses were performed using SAS® version 9.2.

Biomarkers of CTC counts and CXCR4 expression in tumors and CTCs were summarized for the overall study population and by treatment arm. Baseline values were compared between treatment arms using a Mann-Whitney test. The Pearson product moment correlation coefficient for baseline CXCR4 expression in tumor tissue versus CTCs was calculated. Receiver operating characteristic (ROC) curves, AUCs, and Wald 95\% CIs were generated using PROC LOGISTIC to determine optimum biomarker cutoffs (i.e., values that optimize sensitivity plus specificity). PFS and OS were evaluated at 4 and 6 months by optimum biomarker cutoffs using Kaplan-Meier analysis and log-rank test using PROC
LIFETEST, with a Cox proportional hazards regression analysis using PROC PHREG to determine the HR and corresponding $95 \% \mathrm{CI}$.

\section{Results}

\section{Patient characteristics and biomarker levels}

Patient characteristics and demographics were published previously [23]. Of the 94 patients randomized, 90 ED-SCLC patients received treatment with LY2510924 plus CE $(N=47)$ or CE $(N=43)$ and comprised the overall study population. The present exploratory analyses included data from the efficacy population of 89 patients (Table 1): LY2510924 plus CE $(N=47)$ or CE $(N=42)$; data from 1 patient in the $\mathrm{CE}$ arm were excluded due to sub-therapeutic dosing on cycle 1 , day 1 (protocol violation). The CXCR4 detection assay in CTCs became available after the study had started, and 25 of 292 samples had CTC counts without CXCR4 expression evaluation. A total of 15 patient blood samples did not yield evaluable CTC data due to insufficient sample volume, interference substance detected in enriched CTCs, or incorrect sample handling.

There were no statistical differences between treatment arms for baseline tumor CXCR4 expression, CXCR4 expression in CTCs, and CTC counts (Table 1). At baseline, $83 \%$ of the overall study population had $\geq 1 \mathrm{CTCs} / 7.5 \mathrm{~mL}$ blood with a mean of 851.0 and median of 51.0. The baseline $\% \mathrm{CXCR}^{+}$CTCs mean was 23.6 with a median of 18.0. The $\mathrm{CXCR}^{+}$tumor tissue $\mathrm{H}$-score mean was 203.0 with a median of 200.0. For the overall study population, baseline CXCR4 expression in tumor tissue positively correlated with baseline $\% \mathrm{CXCR} 4^{+} \mathrm{CTCs}$ $(r=0.423,95 \%$ CI $=0.174,0.616 ; P=0.001)$ (Supplementary Table S1; Supplementary Fig. S2a).

\section{Prognostic value of biomarker levels for PFS and OS}

The phase II study demonstrated no difference in outcomes between the 2 treatment arms [23]; therefore, the overall study population data were combined to determine optimum cutoffs based on ROC for 6-month PFS (Supplementary Table S2; Supplementary Fig. S2b-f). These optimum cutoff values were: $\mathrm{H}$-score $\geq 210$ for $\mathrm{CXCR} 4^{+}$tumor tissue, CTCs $\geq 6$ / $7.5 \mathrm{~mL}$ blood, and CXCR4 $4^{+} \mathrm{CTCs} \geq 7 \%$, and were evaluated by treatment and visit (Supplementary Table S3) or endpoint (Supplementary Table S4). The AUC values for these 3 optimum cutoffs with sensitivity, specificity, and positive and negative predictive values are shown in Supplementary Table S2. Baseline \%CXCR4 ${ }^{+}$CTCs was the only biomarker with a lower limit of the CI for 
Table 1 Baseline tumor CXCR4 expression by IHC and CTC counts and CXCR4 expression in CTCs (\% CXCR4 ${ }^{+}$CTC) at baseline and post-treatment by treatment arm or overall study population

\begin{tabular}{|c|c|c|c|}
\hline Biomarker & $\begin{array}{l}\mathrm{LY} 2510924+\mathrm{CE} \\
(N=47)\end{array}$ & $\begin{array}{l}\mathrm{CE} \\
(N=42)\end{array}$ & $\begin{array}{l}\text { Total } \\
(N=89)\end{array}$ \\
\hline \multicolumn{4}{|l|}{$\mathrm{CXCR}^{+}{ }^{+}$Tumor Tissue } \\
\hline \multicolumn{4}{|l|}{ Baseline } \\
\hline Patients with evaluable results, $n$ & 36 & 33 & 69 \\
\hline Mean H-score (SD) & $205.8(80.4)$ & $199.8(85.2)$ & $203.0(82.2)$ \\
\hline Median H-score (range) & $200.0(20,300)$ & $200.0(5,300)$ & $200.0(5,300)$ \\
\hline$P$-value* & & & 0.808 \\
\hline \multicolumn{4}{|l|}{ CTC Count } \\
\hline \multicolumn{4}{|l|}{ Baseline } \\
\hline Patients with evaluable results, $n$ & 42 & 36 & 78 \\
\hline Mean (SD) & $615.5(1419.4)$ & $1125.7(3865.7)$ & $851.0(2816.2)$ \\
\hline Median (range) & $20.5(0,7153)$ & $98.5(0,21,428)$ & $51.0(0,21,428)$ \\
\hline$P$-value* & & & 0.262 \\
\hline Patients with CTC, $n / N(\%)$ & $33 / 42(78.6)$ & $32 / 36(88.9)$ & $65 / 78(83.3)$ \\
\hline \multicolumn{4}{|l|}{ Cycle 1 , day 7} \\
\hline Patients with evaluable results, $n$ & 32 & 30 & 62 \\
\hline Mean (SD) & $118.2(461.3)$ & $57.4(156.1)$ & $88.8(347.3)$ \\
\hline Median (range) & $4.0(0,2624)$ & $2.5(0,594)$ & $2.5(0,2624)$ \\
\hline Patients with CTC, $n / N(\%)$ & 23/32 (71.9) & $19 / 30(63.3)$ & $42 / 62(67.7)$ \\
\hline \multicolumn{4}{|l|}{ Cycle 2, day 1} \\
\hline Patients with evaluable results, $n$ & 34 & 27 & 61 \\
\hline Mean (SD) & $70.6(209.3)$ & $25.1(79.9)$ & $50.5(165.5)$ \\
\hline Median (range) & $1.5(0,875)$ & $0.0(0,406)$ & $0.0(0,875)$ \\
\hline Patients with CTC, $n / N(\%)$ & $18 / 34(52.9)$ & $12 / 27(44.4)$ & $30 / 61(49.2)$ \\
\hline \multicolumn{4}{|l|}{ 30-day follow-up } \\
\hline Patients with evaluable results, $n$ & 29 & 25 & 54 \\
\hline Mean (SD) & $128.5(476.3)$ & $5.2(18.1)$ & $71.4(351.9)$ \\
\hline Median (range) & $0.0(0,2008)$ & $0.0(0,87)$ & $0.0(0,2008)$ \\
\hline Patients with CTC, $n / N(\%)$ & $14 / 29(48.3)$ & $9 / 25(36.0)$ & $23 / 54(42.6)$ \\
\hline \multicolumn{4}{|l|}{$\% \mathrm{CXCR}^{+} \mathrm{CTCs}$} \\
\hline \multicolumn{4}{|l|}{ Baseline } \\
\hline Patients with evaluable results, $n$ & 37 & 33 & 70 \\
\hline Mean (SD) & $21.0(26.3)$ & $26.6(24.7)$ & $23.6(25.5)$ \\
\hline Median (range) & $10.9(0.0,100.0)$ & $21.1(0.0,100.0)$ & $17.8(0.0,100.0)$ \\
\hline$P$-value* & & & 0.182 \\
\hline \multicolumn{4}{|l|}{ Cycle 1 , day 7} \\
\hline Patients with evaluable results, $n$ & 29 & 27 & 56 \\
\hline Mean (SD) & $0.1(0.2)$ & $9.2(17.5)$ & $4.4(12.9)$ \\
\hline Median (range) & $0.0(0.0,1.0)$ & $0.0(0.0,58.3)$ & $0.0(0.0,58.3)$ \\
\hline \multicolumn{4}{|l|}{ Cycle 2, day 1} \\
\hline Patients with evaluable results, $n$ & 33 & 24 & 57 \\
\hline Mean (SD) & $7.9(15.0)$ & $10.6(19.7)$ & $9.1(17.0)$ \\
\hline Median (range) & $0.0(0.0,50.0)$ & $0.0(0.0,66.6)$ & $0.0(0.0,66.7)$ \\
\hline \multicolumn{4}{|l|}{ 30-day follow-up } \\
\hline Patients with evaluable results, $n$ & 29 & 23 & 52 \\
\hline Mean (SD) & $4.1(13.2)$ & $8.7(28.8)$ & $6.1(21.4)$ \\
\hline Median (range) & $0.0(0.0,50.0)$ & $0.0(0.0,100.0)$ & $0.0(0.0,100.0)$ \\
\hline
\end{tabular}

CE Carboplatin-etoposide, CTC Circulating tumor cell, CXCR4 Chemokine (C-X-C motif) receptor 4, IHC Immunohistochemistry, $N$ number of patients, $n$ Number of patients in a category, $S D$ Standard deviation $* P$-value from 2-sample, 2-sided Mann-Whitney of null hypothesis that baseline values were same for treatment arms 
a

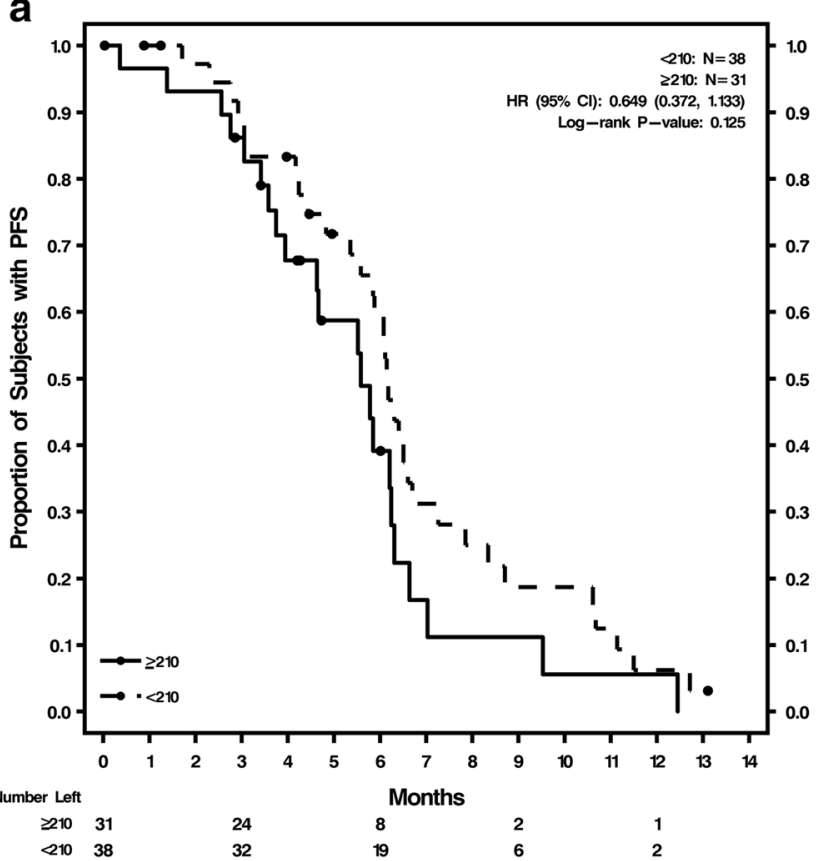

C

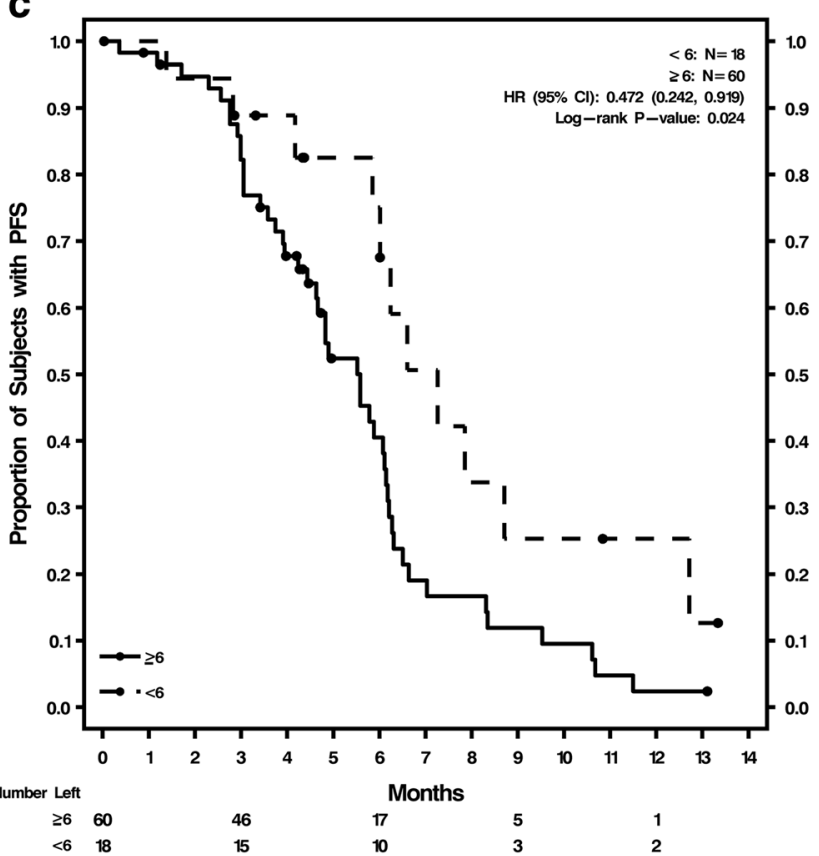

Fig. 1 Kaplan-Meier curves for PFS and OS in the overall patient population by biomarker levels a Baseline CXCR4 expression in tumor tissue $\mathrm{H}$-score $\geq 210$ and $<210$ for PFS; $\mathbf{b}$ Baseline CXCR4 expression in tumor tissue $\mathrm{H}$-score $\geq 210$ and $<210$ for OS; c Baseline CTC counts $\geq 6$ and $<6$ for PFS; $\mathbf{d}$ Baseline CTC counts $\geq 6$ and $<6$ for OS; e Cycle 2, day

AUC $>0.5($ AUC $=0.702 ; C I=0.577,0.828$; $P=0.011)$ and a significant $P<0.05$.

Kaplan-Meier survival estimates and Cox regression analyses were done using optimum cutoffs of these 3 biomarkers, comparing patients above and below the cutoffs in the overall

\section{b}

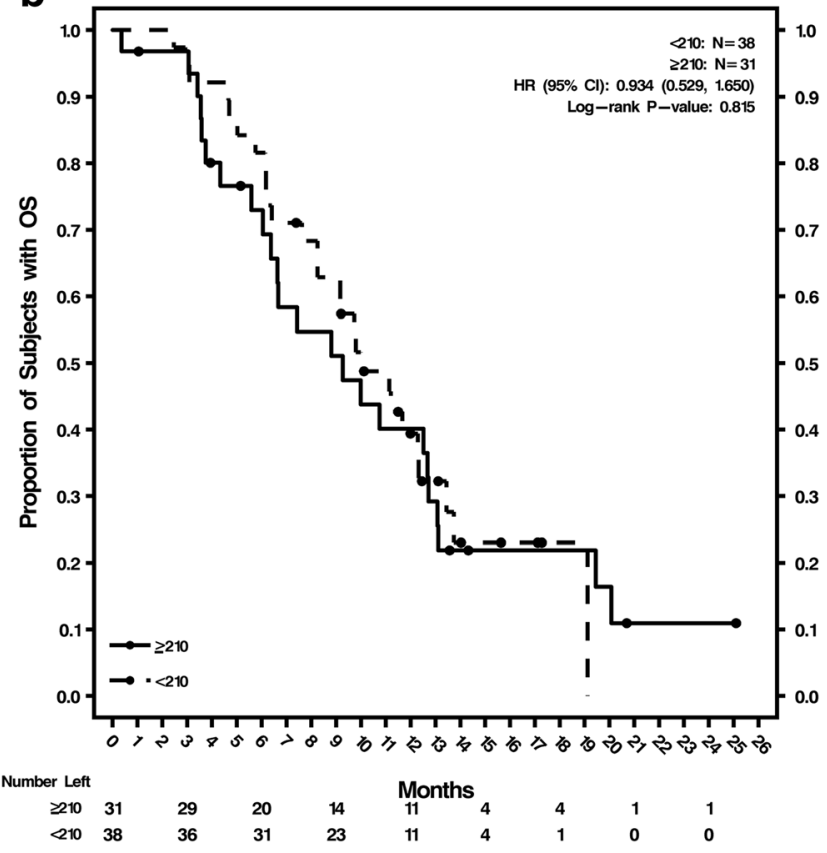

d

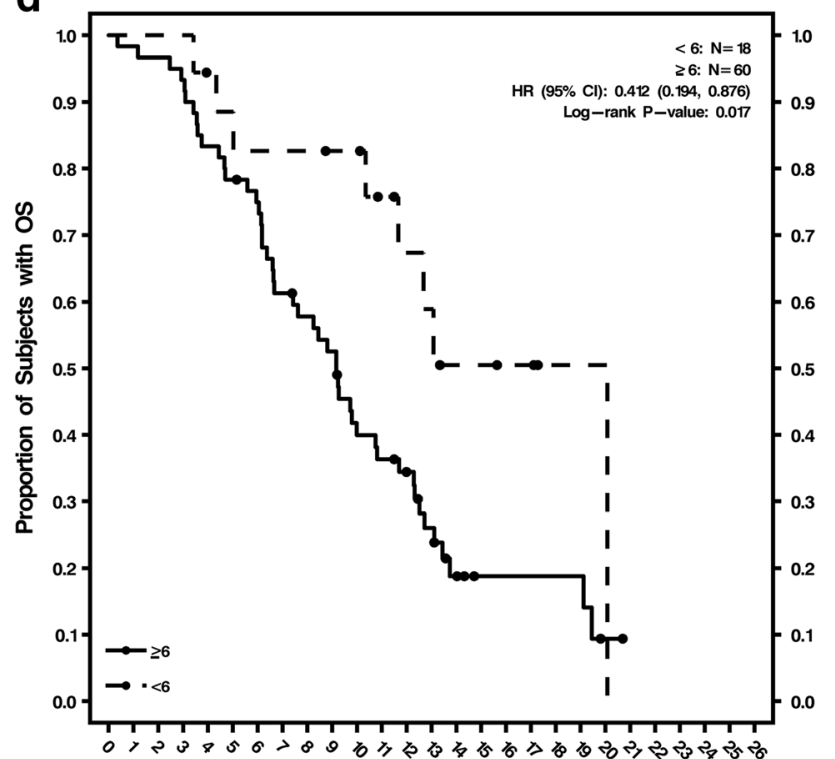

Number Left

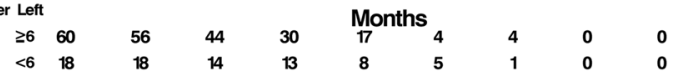

1 CTC counts $\geq 6$ and $<6$ for PFS; f Cycle 2 , day 1 CTC counts $\geq 6$ and $<6$ for OS; $\mathbf{g}$ Baseline $\% \mathrm{CXCR} 4^{+} \mathrm{CTCs} \geq 7 \%$ and $<7 \%$ for PFS; $\mathbf{h}$ Baseline $\% \mathrm{CXCR}^{+} \mathrm{CTCs} \geq 7 \%$ and $<7 \%$ for OS; i Cycle 2 , day $1 \% \mathrm{CXCR} 4^{+}$ CTCs $\geq 7 \%$ and $<7 \%$ for PFS; $\mathbf{j}$ Cycle 2 , day $1 \%$ CXCR $4^{+}$CTCs $\geq 7 \%$ and $<7 \%$ for OS

study population (Fig. 1a-j; Supplementary Table S5). The analyses were conducted using the cutoffs at baseline and at cycle 2, day 1 for both PFS and OS. Baseline tumor tissue CXCR4 expression was not prognostic of either PFS or OS in the overall study population. The Kaplan-Meier curves 

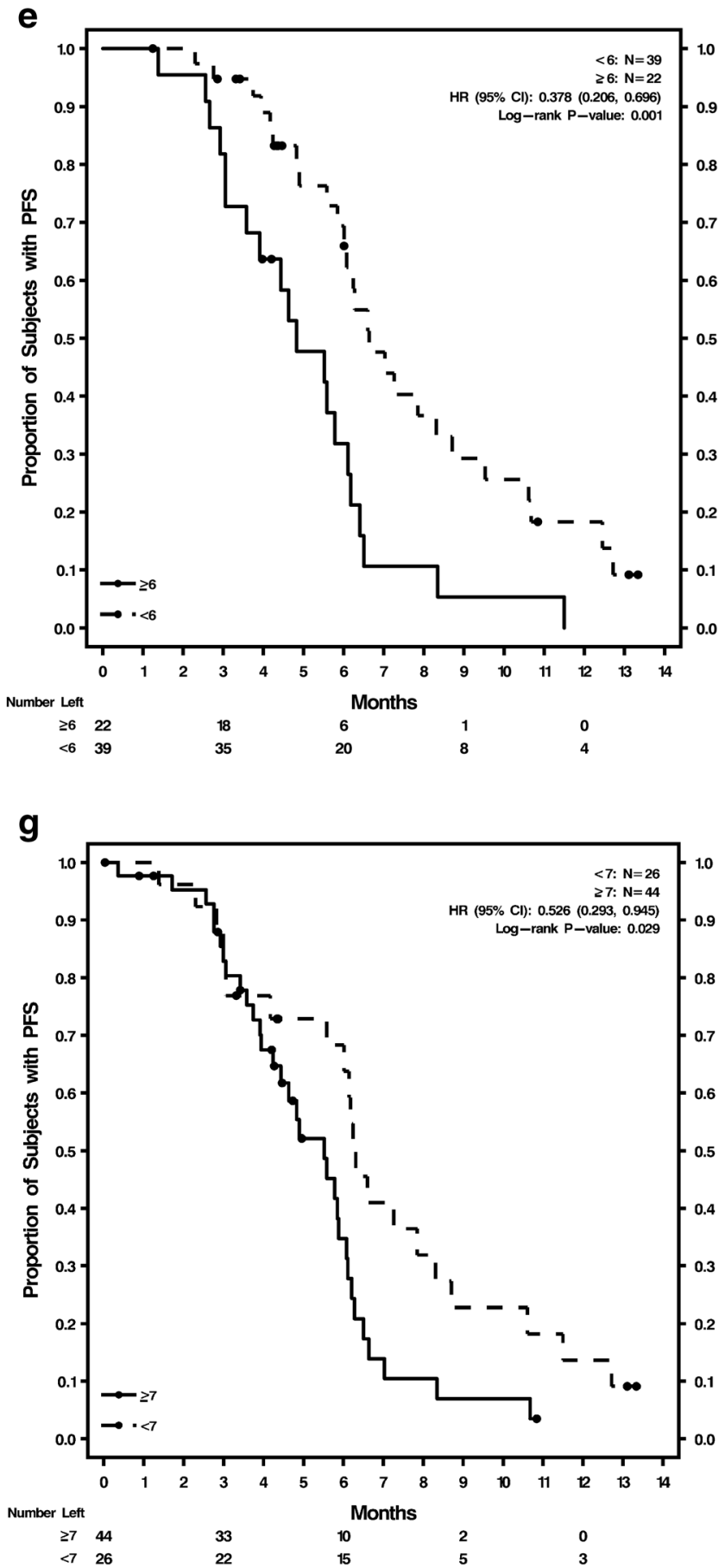

Fig. 1 (continued)

(Fig. 1a, b) were not well separated for $\mathrm{H}$-scores of $\geq 210$ versus $<210$. CTCs $\geq 6$ were prognostic of shorter PFS and OS at baseline and at cycle 2, day 1: CTCs at baseline were significant indicators for PFS $(P=0.024)$ and OS $(P=0.017)$, and CTCs at cycle 2, day 1 were significant indicators for PFS $(P=0.001)$ and $\mathrm{OS}(P=0.001)$. Kaplan-Meier curves for PFS and OS were well separated for CTCs $\geq 6$ versus $<6$ at baseline (Fig. 1c, d) and cycle 2, day 1 (Fig. 1e, f). Baseline CXCR4 ${ }^{+}$ CTCs $\geq 7 \%$ was prognostic of shorter PFS $(P=0.029)$ but not
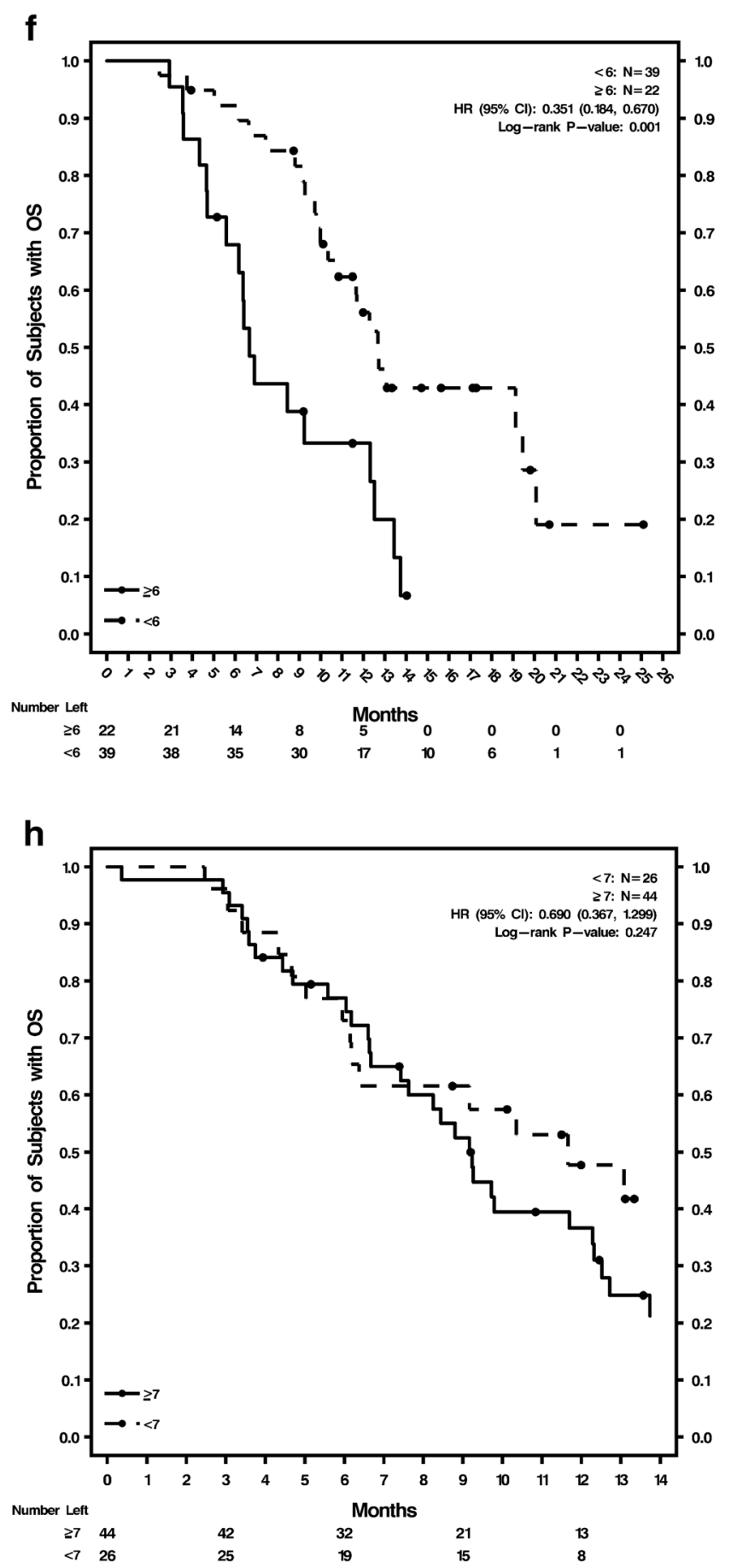

OS (Supplementary Table S5; Fig. 1g, h). CXCR $4^{+}$CTCs $\geq 7 \%$ at cycle 2, day 1 was not prognostic of either PFS or OS (Supplementary Table S5; Fig. 1i, j).

\section{Predictive value of biomarker levels for PFS and OS} by treatment arm

The optimum cutoffs for the 3 biomarkers (H-score $\geq 210$ for $\mathrm{CXCR}^{+}$tumor tissue, CTCs $\geq 6$, and CXCR $4^{+}$CTCs $\geq 7 \%$ ) 


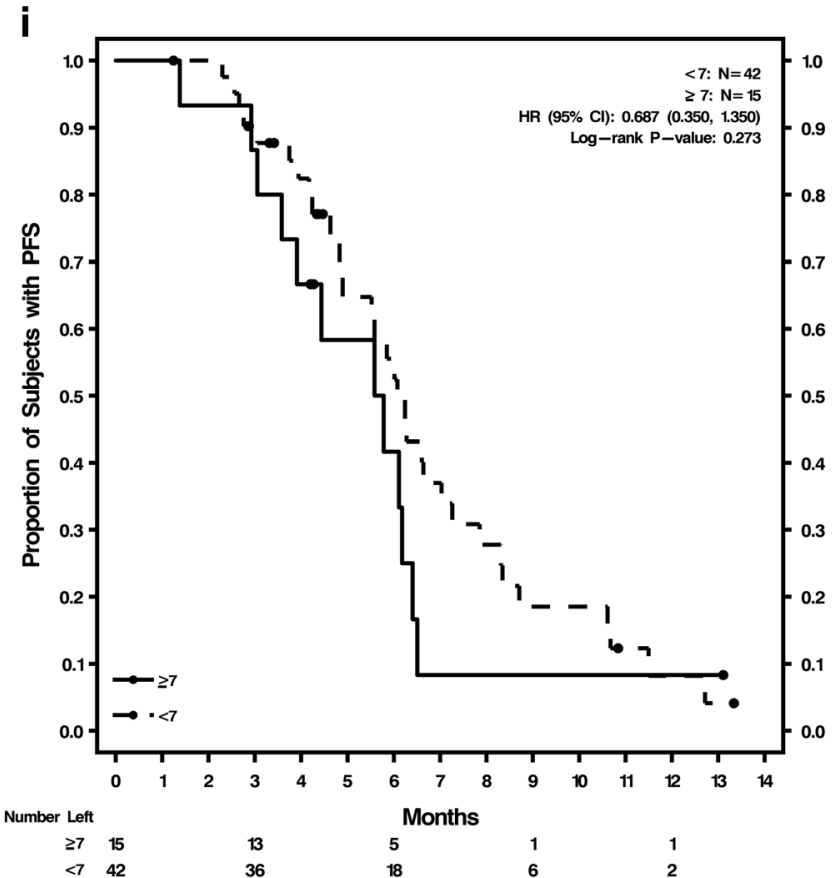

Fig. 1 (continued)

were applied to survival analyses by treatment arm. KaplanMeier survival estimates (in months) and HR values for PFS and OS at 4 and 6 months are shown by treatment arm in Supplementary Table S4. All 3 biomarkers were analyzed at baseline, and CTCs and \%CXCR $4^{+}$CTCs at cycle 2 , day 1 . None of the 3 biomarkers at their respective optimum cutoffs was predictive of treatment response. Representative KaplanMeier curves are shown in Fig. 2, including PFS for patients with baseline CTCs $\geq 6$ and PFS for patients with baseline CXCR $4^{+}$CTCs $\geq 7 \%$, by treatment arm (Fig. 2a, b). The Kaplan-Meier curves were separated in the first 4 months of treatment, but were no longer separated after approximately 4-5 months. Per study design, patients were on treatment for a maximum of six 21-day cycles, which was approximately 4 months. An analysis was conducted for patients with both baseline CTCs $\geq 6$ and CXCR $4^{+}$CTCs $\geq 7 \%$, considered a higher-risk group, to evaluate whether a combined elevation of these biomarkers may be predictive of treatment response as measured as PFS and OS. Kaplan-Meier survival curves and HR values show that this combination of elevated baseline biomarkers was not predictive of PFS (Table 2; Fig. 2c) or OS (data not shown).

\section{Discussion}

In a phase II study (NCT01439568 [22]) of LY2510924, a cyclic peptide that blocks the binding of the ligand SDF-1 (CXCL12) to CXCR4 [16, 21], there was no difference in median PFS for patients with ED-SCLC treated with

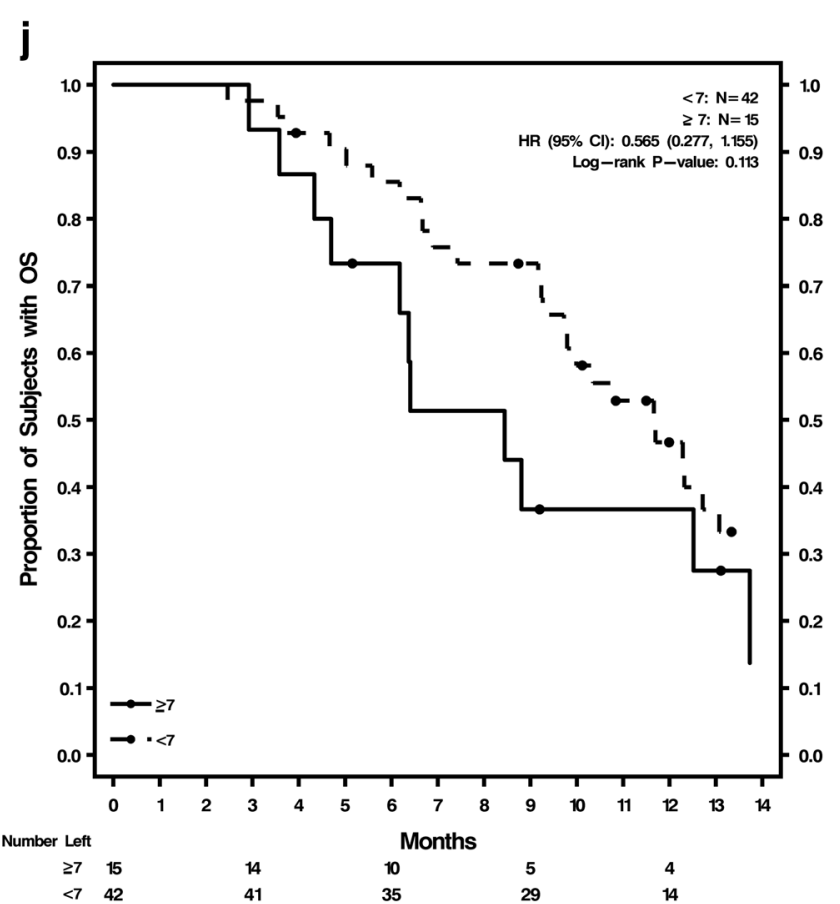

LY2510924 plus CE and CE [23]. We conducted post-hoc exploratory analyses to evaluate the prognostic value of CTC counts and CXCR4 expression in both CTCs and tumor tissue in the overall study population, the predictive value of these biomarkers for treatment response to LY2510924 plus $\mathrm{CE}$ versus $\mathrm{CE}$ alone, and the correlation of CXCR4 expression in CTCs and tumors. These exploratory analyses were done on a limited dataset with no adjustments for multiplicity, and the results should be considered as hypotheses that need further testing. The proportion of patients $(83 \%)$ in our study with $\geq 1 \mathrm{CTC} / 7.5 \mathrm{~mL}$ blood at baseline was similar to Normanno et al. [26]. The median CTC count at baseline in our study is comparable to reports in the literature for SCLC (Hou et al. [8], Huang et al. [14], and Normanno et al. [26]).

The CELLSEARCH system has been used to detect CTCs in various tumor types, including SCLC, making CTC counts or characterization a useful biomarker to establish cutoffs [9, $12,14]$. In the present analyses using CELLSEARCH, an optimum cutoff of $\geq 6 \mathrm{CTCs} / 7.5 \mathrm{~mL}$ blood at baseline and post-treatment (cycle 2, day 1) was prognostic of shorter PFS and OS. There were $77 \%$ and $36 \%$ of the patients in this study with baseline and cycle 2 , day 1 CTC counts $\geq 6$, respectively. Other studies have defined variable CTC cutoffs that demonstrated prognostic value for treatment outcomes: 50 CTCs/7.5 mL by Hou et al. [9], 8 CTCs/7.5 mL by Naito et al. [12], 2 CTCs/7.5 mL by both Hiltermann et al. [10] and $\mathrm{Wu}$ et al. [27], $5 \mathrm{CTCs} / 7.5 \mathrm{~mL}$ by Cheng et al. [28], and 282 CTCs/7.5 mL by Normanno et al. [26]. In our analyses, a cutoff of $\geq 6$ CTCs was prognostic of both PFS and OS but was not predictive of 4- or 6-month PFS for treatment with 

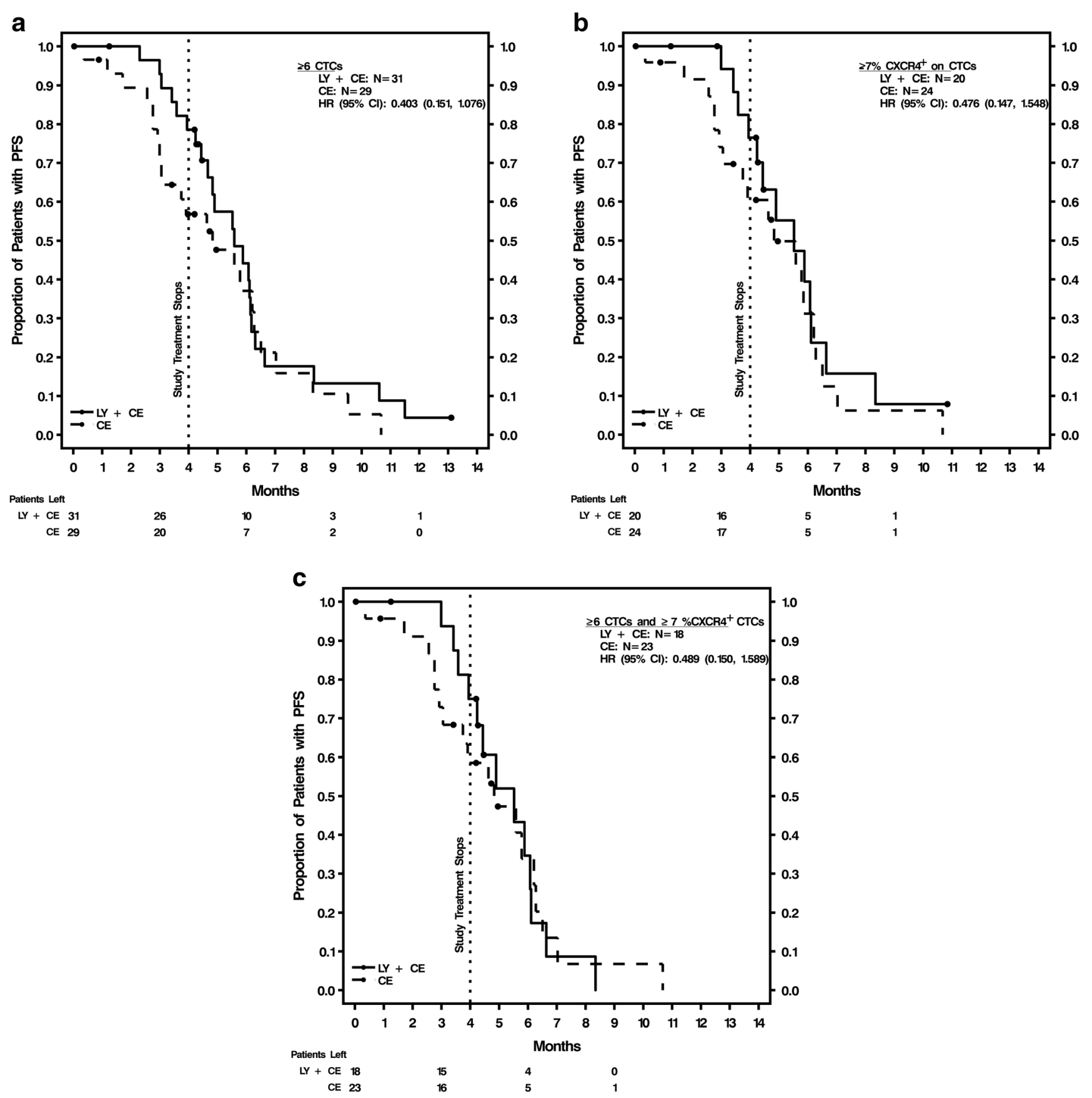

Fig. 2 Kaplan-Meier curves for PFS by treatment arm and biomarker levels. a Baseline $\geq 6$ CTCs; $\mathbf{b}$ Baseline $\geq 7 \%$ CXCR $4^{+}$CTCs; $\mathbf{c}$ Baseline $\geq 7 \% \mathrm{CXCR}^{+}{ }^{+} \mathrm{CTCs}$ and $\geq 6 \mathrm{CTCs}$. $C E$ carboplatin-etoposide, $C I$ confidence interval, CTCs circulating tumor cells, CXCR4 chemokine

(C-X-C motif) receptor $4, H R$ hazard ratio at 4 months (end of treatment), $L Y$ LY2510924, $N$ number of patients, $O S$ overall survival, PFS progression-free survival

LY2510924 plus CE versus CE. To our knowledge, this was the first analysis of CXCR4 expression in CTCs in SCLC, and a comparison of CXCR4 expression in tumor and CTCs (which may derive from the primary tumor or metastatic sites) showed a weak positive correlation. CXCR4 baseline overexpression in tumor $(\geq 210 \mathrm{H}$-score $)$ was not prognostic of shorter PFS or OS in patients with ED-SCLC. Baseline overexpression of CXCR4 in CTCs $\left(\geq 7 \%\right.$ CXCR $4^{+}$CTCs) was prognostic of shorter PFS, but not OS. Post-treatment (cycle 2, day 1) overexpression of CXCR4 in CTCs $\left(\geq 7 \%\right.$ CXCR4 ${ }^{+}$ CTCs) was not prognostic of PFS or OS.

In both treatment arms, we observed median CTC counts and median \%CXCR4+ CTCs decreases from baseline. Our data showed that if CTCs are $\geq 6$ at cycle 2 , day 1 it is a very strong prognostic biomarker of poor survival outcome (PFS and OS). Our data are consistent with several reports showing 
Table 2 Predictive value of combined elevated baseline markers for PFS (4 months or 6 months) by treatment arm

\begin{tabular}{llll}
\hline & $\begin{array}{l}\text { LY2510924 + CE } \\
(N=18)\end{array}$ & $\begin{array}{l}\text { CE } \\
(N=23)\end{array}$ & $\begin{array}{l}\text { Total } \\
(N=41)\end{array}$ \\
\hline $\begin{array}{l}\text { Baseline } \geq 7 \% \text { CXCR4 }+ \text { CTCs and } \geq 6 \text { CTC count } \\
\text { Kaplan-Meier estimate (mos) }\end{array}$ & & & \\
$\quad$ Mean (SD) & $5.3(0.4)$ & $4.9(0.6)$ & $5.0(0.4)$ \\
Median (95\% CI) & $5.5(3.9,6.1)$ & $4.8(2.9,6.2)$ & $4.9(3.9,6.1)$ \\
HR LY2510924 + CE vs CE (95\% CI) & & & $0.94(0.46,1.95)$ \\
$P$-value* & & & 0.872 \\
HR LY2510924 + CE vs CE (95\% CI) 4 mos & & $0.49(0.15,1.59)$ \\
$P$-value through 4 mos* & & 0.223 \\
HR LY2510924 + CE vs CE (95\% CI) 6 mos & & $0.79(0.34,1.85)$ \\
$P$-value through 6 mos* & & 0.588 \\
\hline
\end{tabular}

CE Carboplatin-etoposide, CI Confidence interval, CTCs Circulating tumor cell, HR Hazard ratio, mos Months $* P$-value from a log-rank test that when CTCs are decreased in response to chemotherapy in patients with SCLC, this can serve as a prognostic biomarker. Naito et al. [12] showed that patients with post-treatment CTCs $\geq 8$ had worse outcomes versus CTCs $<8(P=0.0096)$; patients with $\geq 8$ CTCs at baseline also had a worse prognosis versus $<8$ CTCs at baseline $(P=0.0014)$. A study by Hou et al. [9] of patients with SCLC treated with first-line chemotherapy reported a decrease from pre-treatment CTC counts after 1 cycle of chemotherapy, which was an independent prognostic factor, and a higher number of CTCs were associated with shorter OS and PFS. Similarly, Cheng et al. [28] reported that in Chinese patients with ED-SCLC following 2 cycles of chemotherapy, $\geq 5 \mathrm{CTCs} / 7.5 \mathrm{~mL}$ decreases led to significantly shorter median OS versus $<5$ CTCs $/ 7.5 \mathrm{~mL}$ blood $(P<0.0124)$; multivariate analyses showed CTCs was an independent prognostic marker for OS at baseline $(P=0.018)$ and after 2 cycles of chemotherapy $(P=0.0249)$. Overall, CTC counts appear to be a promising prognostic biomarker for ED-SCLC; collecting CTCs from peripheral blood as a liquid biopsy, amenable to repeat sampling, circumvents a need for invasive, inaccessible, solid biopsies in this patient population.

CXCR4 is overexpressed in a variety of human cancers including breast, lung, kidney, colon, ovarian, and brain [29]. The landscape of clinical development of anti-CXCR4 therapies is emerging, and, at present, the prognostic role of CXCR4 overexpression as a biomarker across different tumors is unclear. This overexpression can correlate with increased risk for recurrence and poor survival, depending on tumor type and outcomes studied. Minamiya et al. [30] reported that in patients with adenocarcinoma of the lung, higher levels of tumor cell CXCR4 expression are prognostic of better outcomes. In contrast, $\mathrm{Li}$ et al. [31] reported that CXCR4 co-expression with urokinase-type plasminogen activator receptor predicts worse prognosis of SCLC patients. In a metaanalysis, the expression of CXCR4 was an independent prognostic factor for lower survival and increased metastasis in
SCLC [32]. In our analysis, despite a weak positive correlation between $\mathrm{CXCR}^{+}{ }^{+}$tumor and $\mathrm{CXCR}^{+}{ }^{+} \mathrm{CTCs}$, CXCR4 overexpression in tumor was not a prognostic factor for survival outcomes. We analyzed CXCR4 expression in CTCs by treatment arm, and Kaplan-Meier curves showed separation in PFS between treatment arms through the 4-month course of therapy, which did not reach statistical significance, for $\geq 6$ CTCs or $\geq 7 \% \mathrm{CXCR}^{+}$CTCs (Fig. 2a, b). This separation was not observed at 6 months. Since no differences in clinical endpoints were observed between treatment arms [23], data from both arms were combined for a larger sample size to evaluate biomarker prognostic value. This is a potential limitation of the exploratory post-hoc analyses; a prospective study is required to confirm CTC counts as prognostic of outcomes. It is notable that the combined sample size of our study $(N=89)$ is greater than those reported in several prior studies: 38 patients in Hiltermann et al. [10], and 24 patients in Naito et al. [12].

Our finding, that CXCR4 overexpression in tumors is not prognostic of outcomes in SCLC, contrasts with several recent meta-analyses of biomarker levels and lung cancer clinical outcomes. Liang et al. [32] showed high-level CXCR4 tumor expression (2037 patients) is related to poor prognosis in lung cancer patients, and Zhao et al. [33] showed that CXCR4 overexpression (11,032 patients) predicts unfavorable OS in lung cancer. Our analyses represent the first report we are aware of measuring CXCR4 expression in both tumor and CTCs, using the CELLSEARCH® platform. One previous report from Reckamp et al. [34] showed that low coexpression of CXCR4 and pan-cytokeratin in CTCs was prognostic of improved OS in NSCLC, based on flow cytometry analysis of peripheral blood from 16 patients; CXCR4 expression in NSCLC tumors was documented, but not further analyzed in addition to CTCs. Our observation, that elevated baseline CXCR4 expression in CTCs ( $\geq 7 \%$ CXCR4 $\left.{ }^{+} \mathrm{CTCs}\right)$ is prognostic of worse PFS in ED-SCLC, whereas CXCR4 
overexpression in tumor is not, has several possible explanations beyond study design. CTCs may better represent the cells shed by primary and metastatic tumors versus tumor tissue biopsied from a single site. CTC samples drawn immediately prior to start of the study may better represent disease status versus tumor biopsy that may have been obtained less proximal to start of the study. Additional prospective study is required for confirmation and clarity on $\mathrm{CXCR} 4^{+} \mathrm{CTCs}$ as a prognostic biomarker. Lastly, several isoforms of CXCR4 have been identified in cancer cell lines, including alternate splice variants $[35,36]$. Our study did not differentiate between isoforms of CXCR4 detected in biopsied tumor and blood CTCs, the clinical relevance of which is not known.

In summary, positive CXCR4 expression in tumor tissue was not significantly prognostic of survival in 89 patients with ED-SCLC in the present analysis. Neither baseline CTC counts or CXCR4 expression in tumor tissue or CTCs were predictive of treatment response for CXCR4 peptide antagonist LY2510924 plus CE versus CE alone. However, in general, CTC enumeration and CXCR4 expression in CTCs are promising prognostic biomarkers for ED-SCLC at baseline and post-treatment, as evidenced in the literature. The feasibility of conducting biomarker, prognostic, and predictive analyses in future SCLC studies is facilitated by peripheral blood CTC collection as a liquid biopsy, which has the potential to replace technically difficult solid biopsies in this patient population.

Acknowledgements The authors thank the patients, their families, the study staff, and the investigators at the study sites who were involved in conducting the clinical study in the collection of samples for circulating tumor cells. The authors would like to acknowledge Sharad Wankhade, $\mathrm{PhD}$, Jennifer Bodie, $\mathrm{PhD}$, and Noelle Gasco, employees of inVentiv Health Clinical, for writing and editorial support, which was funded by Eli Lilly and Company.

\section{Compliance with ethical standards}

Disclosure of potential conflicts of interest J.R. Stille, S.B. Yan, S. Roberson, J. Polzer, E. Raddad, V.L. Peek, S.R. Wijayawardana, and S.L. Um are employees and shareholders of Eli Lilly and Company.

S. Gross, M.C. Connelly, C. Morano, M. Repollet, R. Sanders, K. Baeten, and D. D'Haese are employees of Janssen Diagnostics and shareholders of Johnson and Johnson Company.

S. Gross and C. Morano receive royalties from Johnson and Johnson Company.

D.R. Spigel receives no compensation as an advisor to Bristol-Myers Squibb, Genentech/Roche, Novartis, and Pfizer and serves on a data safety monitoring board for Merck.

No conflict of interest was reported by R. Salgia, M. McCleod, R.W. Weaver, or A. Flynt.

Funding This study was funded by Eli Lilly and Company.

Ethical approval All procedures performed in studies involving human participants were in accordance with the ethical standards of the institutional and/or national research committee and with the 1964
Declaration of Helsinki and its later amendments or comparable ethical standards.

Informed consent Informed consent was obtained from all individual participants included in the study. The informed consent form was signed by the patient and physician prior to study procedures in accordance with International Conference on Harmonization guidelines on Good Clinical Practice.

Open Access This article is distributed under the terms of the Creative Commons Attribution 4.0 International License (http:// creativecommons.org/licenses/by/4.0/), which permits unrestricted use, distribution, and reproduction in any medium, provided you give appropriate credit to the original author(s) and the source, provide a link to the Creative Commons license, and indicate if changes were made.

\section{References}

1. American Cancer Society (2016) Lung cancer (small cell) http://www. cancer.org/acs/groups/cid/documents/webcontent/003116-pdf.pdf. Accessed 11 Aug 2016

2. Reck M, Thatcher N, Smit EF et al (2012) Baseline quality of life and performance status as prognostic factors in patients with extensive-stage disease small cell lung cancer treated with pemetrexed plus carboplatin vs. etoposide plus carboplatin. Lung Cancer 78:276-281

3. American Cancer Society (2016) Cancer facts \& figures 2016. American Cancer Society, Atlanta

4. Niell HB (2001) Extensive stage small cell lung cancer. Curr Treat Options in Oncol 2:71-76

5. Wang H, Xie F, Hu Z, Chen L (2015) Elevated expression of CXCR4 and correlation with clinicopathological features and prognosis of non-small cell lung cancer patients: a meta-analysis. Genet Mol Res 14:17893-17903

6. Hodgkinson CL, Morrow CJ, Li Y et al (2014) Tumorigenicity and genetic profiling of circulating tumor cells in small-cell lung cancer. Nat Med 20:897-903

7. Bevilacqua S, Gallo M, Franco R et al (2009) A "live" biopsy in a small-cell lung cancer patient by detection of circulating tumor cells. Lung Cancer 65:123-125

8. Hou JM, Greystoke A, Lancashire L et al (2009) Evaluation of circulating tumor cells and serological cell death biomarkers in small cell lung cancer patients undergoing chemotherapy. Am J Pathol 175:808-816

9. Hou JM, Krebs MG, Lancashire L et al (2012) Clinical significance and molecular characteristics of circulating tumor cells and circulating tumor microemboli in patients with small-cell lung cancer. J Clin Oncol 30:525-532

10. Hiltermann TJ, Pore MM, van den Berg A et al (2012) Circulating tumor cells in small-cell lung cancer: a predictive and prognostic factor. Ann Oncol 23:2937-2942

11. Igawa S, Gohda K, Fukui T et al (2014) Circulating tumor cells as a prognostic factor in patients with small cell lung cancer. Oncol Lett 7:1469-1473

12. Naito T, Tanaka F, Ono A et al (2012) Prognostic impact of circulating tumor cells in patients with small cell lung cancer. J Thorac Oncol 7:512-519

13. Cheng Y, Liu XQ, Fan Y et al (2016) Circulating tumor cell counts/ change for outcome prediction in patients with extensive-stage small-cell lung cancer. Future Oncol 12:789-799

14. Huang CH, Wick JA, Sittampalam GS et al (2014) A multicenter pilot study examining the role of circulating tumor cells as a blood- 
based tumor marker in patients with extensive small-cell lung cancer. Front Oncol 4:271

15. Burger M, Glodek A, Hartmann T et al (2003) Functional expression of CXCR4 (CD184) on small-cell lung cancer cells mediates migration, integrin activation, and adhesion to stromal cells. Oncogene 22:8093-8101

16. Galsky MD, Vogelzang NJ, Conkling P et al (2014) A phase I trial of LY2510924, a CXCR4 peptide antagonist, in patients with advanced cancer. Clin Cancer Res 20:3581-3588

17. Kijima T, Maulik G, Ma PC et al (2002) Regulation of cellular proliferation, cytoskeletal function, and signal transduction through CXCR4 and c-kit in small cell lung cancer cells. Cancer Res 62: 6304-6311

18. Hartmann TN, Burger JA, Glodek A, Fujii N, Burger M (2005) CXCR4 chemokine receptor and integrin signaling co-operate in mediating adhesion and chemoresistance in small cell lung cancer (SCLC) cells. Oncogene 24:4462-4471

19. Sethi T, Rintoul RC, Moore SM et al (1999) Extracellular matrix proteins protect small cell lung cancer cells against apoptosis: a mechanism for small cell lung cancer growth and drug resistance in vivo. Nat Med 5:662-668

20. Teicher BA, Fricker SP (2010) CXCL12 (SDF-1)/CXCR4 pathway in cancer. Clin Cancer Res 16:2927-2931

21. Peng SB, Zhang X, Paul D et al (2015) Identification of LY2510924, a novel cyclic peptide CXCR4 antagonist that exhibits antitumor activities in solid tumor and breast cancer metastatic models. Mol Cancer Ther 14:480-490

22. ClinicalTrials.gov. A study of LY2510924 in patients with extensive-stage small cell lung carcinoma. https://clinicaltrials. gov/ct2/show/NCT01439568. Accessed 12 Jan 2016

23. Spigel DR, Weaver RW, McCleod M et al (2014) Phase II study of carboplatin/etoposide plus LY2510924, a CXCR4 peptide antagonist, versus carboplatin/etoposide in patients with extensive-stage small cell lung cancer (SCLC). Ann Oncol 25(suppl 4):iv511iv516 abstr $1472 \mathrm{P}$

24. Salgia R, Weaver RW, McCleod M et al (2015) Evaluation of CXCR4 expression on tumor and circulating tumor cells (CTCs) as predictive response marker for CXCR4 antagonist LY2510924 in combination with carboplatin-etoposide in extensive-disease small cell lung cancer (ED-SCLC). J Clin Oncol 33 (suppl; abstr 7567)
25. Paoletti C, Muñiz MC, Thomas DG et al (2015) Development of circulating tumor cell-endocrine therapy index in patients with hormone receptor-positive breast cancer. Clin Cancer Res 21:24872498

26. Normanno N, Rossi A, Morabito A et al (2014) Prognostic value of circulating tumor cells' reduction in patients with extensive smallcell lung cancer. Lung Cancer 85:314-319

27. Wu C, Hao H, Li L et al (2009) Preliminary investigation of the clinical significance of detecting circulating tumor cells enriched from lung cancer patients. J Thorac Oncol 4:30-36

28. Cheng Y, Liu XQ, Fan YL et al (2014) Clinical value of circulating tumor cells in monitoring treatment response in Chinese patients with small cell lung cancer. Ann Oncol 25(suppl 4):iv511-iv516 abstr 14640

29. Balkwill F (2004) The significance of cancer cell expression of the chemokine receptor CXCR4. Semin Cancer Biol 14:171-179

30. Minamiya $\mathrm{Y}$, Saito $\mathrm{H}$, Takahashi $\mathrm{N}$ et al (2010) Expression of the chemokine receptor CXCR4 correlates with a favorable prognosis in patients with adenocarcinoma of the lung. Lung Cancer 68:466471

31. Li Y, Shen Y, Miao Y, Luan Y, Sun B, Qiu X (2014) Co-expression of uPAR and CXCR4 promotes tumor growth and metastasis in small cell lung cancer. Int J Clin Exp Pathol 7:3771-3780

32. Liang JX, Gao W, Liang Y, Zhou XM (2015) Chemokine receptor CXCR4 expression and lung cancer prognosis: a meta-analysis. Int J Clin Exp Med 8:5163-5174

33. Zhao H, Guo L, Zhao H, Zhao J, Weng H, Zhao B (2014) CXCR4 over-expression and survival in cancer: a system review and metaanalysis. Oncotarget 6:5022-5040

34. Reckamp KL, Figlin RA, Burdick MD, Dubinett SM, Elashoff RM, Strieter RM (2009) CXCR4 expression on circulating pancytokeratin positive cells is associated with survival in patients with advanced non-small cell lung cancer. BMC Cancer. doi:10.1186 /1471-2407-9-213

35. Kubic JD, Lui JW, Little EC et al (2015) PAX3 and FOXD3 promote CXCR4 expression in melanoma. J Biol Chem 290:2190121914

36. Carlisle AJ, Lyttle CA, Carlisle RY, Maris JM (2009) CXCR4 expression heterogeneity in neuroblastoma cells due to ligandindependent regulation. Mol Cancer. doi:10.1186/1476-4598-8-126 